OPEN ACCESS

Edited by:

Allan V. Kalueff,

Saint Petersburg State University,

Russia

Reviewed by:

Peter A. Bos,

Utrecht University, Netherlands

Darya Meshalkina,

Saint Petersburg University, Russia

*Correspondence:

Mara Dierssen

mara.dierssen@crg.eu

Rafael de la Torre

rtorre@imim.es

Received: 19 May 2016 Accepted: 26 September 2016 Published: 17 October 2016

Citation:

del Hoyo L, Xicota L, Langohr K, Sánchez-Benavides G, de Sola S,

Cuenca-Royo A, Rodriguez J,

Rodríguez-Morató J, Farré $M$,

Dierssen $M$, de la Torre $R$ and the TESDAD Study Group (2016) VNTR-DAT1 and COMTVal158Met Genotypes Modulate Mental Flexibility and Adaptive Behavior Skills in Down Syndrome.

Front. Behav. Neurosci. 10:193. doi: 10.3389/fnbeh.2016.00193

\section{VNTR-DAT1 and COMTVal158Met Genotypes Modulate Mental Flexibility and Adaptive Behavior Skills in Down Syndrome}

\author{
Laura del Hoyo 1,2, Laura Xicota 1,4,5, Klaus Langohr1,3, Gonzalo Sánchez-Benavides', \\ Susana de Sola'1,4, Aida Cuenca-Royo', Joan Rodriguez', Jose Rodríguez-Moratón 1,5,6, \\ Magí Farré1,2, Mara Dierssen ${ }^{4,5,7 *}$, Rafael de la Torre ${ }^{1,5,6 *}$ and The TESDAD Study Group

\begin{abstract}
1 Integrative Pharmacology and Systems Neuroscience Group, Neurosciences Research Program, IMIM-Hospital del Mar Medical Research Institute, Barcelona, Spain, ${ }^{2}$ Departament de Farmacologia, de Terapèutica i de Toxicologia, Universidad Autónoma de Barcelona, Barcelona, Spain, ${ }^{3}$ Department of Statistics and Operations Research, Universidad Politècnica de Cataluña/BarcelonaTech, Barcelona, Spain, ${ }^{4}$ Cellular \& Systems Neurobiology, Systems Biology Program, Centre for Genomic Regulation, The Barcelona Institute of Science and Technology, Barcelona, Spain, ${ }^{5}$ Department of Experimental and Health Sciences, Universidad Pompeu Fabra, Barcelona, Spain, ${ }^{6}$ CIBER Fisiopatología Obesidad y Nutrición, Instituto
\end{abstract} \\ Salud Carlos III, Madrid, Spain, ${ }^{7}$ CIBER de Enfermedades Raras, Instituto Salud Carlos III, Madrid, Spain
}

Down syndrome (DS) is an aneuploidy syndrome that is caused by trisomy for human chromosome 21 resulting in a characteristic cognitive and behavioral phenotype, which includes executive functioning and adaptive behavior difficulties possibly due to prefrontal cortex (PFC) deficits. DS also present a high risk for early onset of Alzheimer Disease-like dementia. The dopamine (DA) system plays a neuromodulatory role in the activity of the PFC. Several studies have implicated trait differences in DA signaling on executive functioning based on genetic polymorphisms in the genes encoding for the catechol-O-methyltransferase (COMTVal158Met) and the dopamine transporter (VNTRDAT1). Since it is known that the phenotypic consequences of genetic variants are modulated by the genetic background in which they occur, we here explore whether these polymorphisms variants interact with the trisomic genetic background to influence gene expression, and how this in turn mediates DS phenotype variability regarding PFC cognition. We genotyped 69 young adults of both genders with DS, and found that VNTR-DAT1 was in Hardy-Weinberg equilibrium but COMTVal158Met had a reduced frequency of Met allele homozygotes. In our population, genotypes conferring higher DA availability, such as Met allele carriers and VNTR-DAT1 10-repeat allele homozygotes, resulted in improved performance in executive function tasks that require mental flexibility. Met allele carriers showed worse adaptive social skills and self-direction, and increased scores in the social subscale of the Dementia Questionnaire for People with Intellectual Disabilities than Val allele homozygotes. The VNTR-DAT1 was not involved in adaptive behavior or early dementia symptoms. Our results suggest that genetic variants of COMTVal158Met and VNTR-DAT1 may contribute to PFC-dependent cognition, while only COMTVal158Met is involved in behavioral phenotypes of DS, similar to euploid population. 


\section{INTRODUCTION}

Down syndrome (DS) is an aneuploidy syndrome that is most commonly caused by trisomy for human chromosome 21 (HSA21) leading to a cognitive and behavioral phenotype characterized by psychomotor delay, cognitive and behavioral deficits, and high risk for early onset of Alzheimer Disease (AD)-like dementia (Grieco et al., 2015). Executive function and adaptive behavior are impaired in DS adults, with the majority of studies showing specific deficits in working memory, attention, mental flexibility, and inhibitory control (Edgin et al., 2012). These functions are commonly ascribed to the prefrontal cortex (PFC) and frontostriatal networks. The function of these circuits relies heavily on neuromodulation, in particular on dopamine (DA) (Miller and Cohen, 2001) These findings are consistent with structural imaging evidences showing reductions in gray matter volumes in the frontal cortex and cingulate gyrus of DS adults (Rowe et al., 2006). While it is assumed that many of this phenotypic DS features stem from enhanced expression of a set of genes harbored within the HSA21, (Korenberg et al., 1994) there is few information about how genetic variants out of the chromosome 21 would be affected by the aneuploidy genomic context.

In the general population, polymorphisms known to influence DA levels in the PFC, such as VNTR in the gene of the DA transporter (DAT) and Val158Met in the gene of the catechol-O-methyltransferase (COMT), are associated to PFC executive function (Caldú et al., 2007; Witte and Flöel, 2012). The COMTVal158Met polymorphism (rs4680) is a SNP (single nucleotide polymorphism) that causes the substitution of a Valine for a Methionine at position 158/108 (Lachman et al., 1996) and has been shown to affect COMT expression, which is reduced in the Met allelic variant carriers. The DAT1 polymorphism is a VNTR (variable number tandem repeat) of 40 nucleotides that can range from three to eleven repetitions and has been shown to affect expression of DAT, with the 10-repeat allele being associated with lower DAT levels than the 9-repeat allele (van de Giessen et al., 2009; Shumay et al., 2011). Both the Met allelic variant and 10-repeat allele would result in greater availability of DA in the PFC, which may be linked to better PFC-dependent cognitive skills (Meyer-Lindenberg et al., 2005; Wonodi et al., 2009; Barnett et al., 2011), whereas these same alleles had been associated with a disadvantage in adaptive behavior (Mier et al., 2009; Koutsilieri et al., 2014).

In DS human brains, the response to dopaminergic stimulation is depressed in post-mortem cerebral cortex (Lumbreras et al., 2006), and reductions in dopamine and its acidic metabolites (DOPAC and HVA) have been detected in DS fetal and adult brains (Risser et al., 1997; Schneider et al., 1997; Whittle et al., 2007). However, only two studies have explored DA genetic polymorphisms. A family-based analysis revealed significant over-transmission of a DRD4 VNTR allele, which encodes for D4 dopamine receptor for DS (Das Bhowmik et al., 2008). In a second study, the 7-repeat allele of the DRD4 polymorphism was associated to behavioral and executive functions difficulties in children with DS (Mason et al., 2015).
Since it is known that the phenotypic consequences of genetic variants are modulated by the genetic background in which they occur, we here explored whether COMTVal158Met and VNTR$D A T 1$ variants interact with the trisomic genetic background to influence gene expression, and how this in turn mediates DS phenotypes. We hypothesized that COMTVal158Met and VNTR$D A T 1$ polymorphisms may influence cognitive and behavioral skills differentially to euploid population.

\section{MATERIALS AND METHODS}

\section{Subjects}

For this cross-sectional study 87 young adults of both genders with DS were drawn from the baseline exploration of a clinical trial carried out by our research group (TESDAD Study ClinicalTrials.gov Identifier: NCT01699711). Participants were excluded if they had neurological disorders other than DS, relevant medical diseases, co-morbid mental disorders or were under any pharmacological treatment that could interfere with cognitive function. Exclusion criteria also included severe language deficit (significant speech and/or comprehension limitations), behavioral disturbances and/or poor level of collaboration during the assessment. Four subjects were excluded for speech and language comprehension limitations, and two declined to participate. Subject disposition, withdrawals and the composition of the primary analysis population are shown in the CONSORT diagram (Supplementary Figure 1).

In the present study, the genotype analysis was performed in the whole eligible sample $(n=81), 10$ subjects could not be genotyped because an insufficient amount of DNA and 11-repeat allele carriers were excluded of the DAT polymorphism analysis since we only detected two subjects, resulting in a final sample of 69 participants of both genders $(M=35, F=34)$ with any of the DS genetic variations (trisomy $21 ; n=65$, partial trisomy; $n=2$, mosaic; $n=1$ or translocation; $n=1$ ), mean age of 25 years (range $=18-37, S D=4.4$ ) and mean IQ of 45 (range $=40-86$, $S D=8.7)$.

The data of a sample of healthy control Spanish subjects genotyped for COMTVal158Met $[n=93$, mean age $=22.8$ (4.1), sex $=49$ M, 44 F; (Cuyàs et al., 2011)], and VNTR-DAT1 $[n=57$, mean age $=22.7(2.83)$, sex $=57 \mathrm{M}, 0 \mathrm{~F}$; unpublished data] were drawn from a previous study (CEIC-IMAS 99/935/I $2001 / 1226 / \mathrm{I}$ ), and were used to assess whether the genotype frequency in the TESDAD DS population was in equilibrium.

\section{Genotype Analysis}

Genomic DNA was extracted from the peripheral blood leukocytes of all the participants using Flexi Gene DNA kit (Qiagen Iberia, S.L., Spain) according to the manufacturer instructions. COMTVal158Met allelic variants were determined as previously described (Verdejo-García et al., 2013). VNTR polymorphism of VNTR-DAT1 was evaluated by PCR using the following primers $5^{\prime}$ TGTGGTGTAGGGAACGGCCTGAG$3^{\prime}$ (forward) and 5'CTTCCTGGAGGTCACGGCTCAAGG-3' (reverse), the reaction conditions for this experiment were the following: 1X PCR Amplification buffer and 1X PCR 
Enhancer solution (Invitrogen, Carlsbad, CA), $1.5 \mathrm{mM} \mathrm{MgSO} 4$, $0.2 \mathrm{mM}$ dNTPs, $0.2 \mu \mathrm{mol}$ of each primer, $1.75 \mathrm{U}$ of Taq DNA polymerase (Invitrogen), and approximately $50 \mathrm{ng}$ of genomic DNA as template. The reaction was carried out on the following conditions: an initial denaturation of $5 \mathrm{~min}$. at $95^{\circ} \mathrm{C}$, followed by 50 cycles as follows: $30 \mathrm{~s}$ denaturation at $95^{\circ} \mathrm{C}, 30 \mathrm{~s}$ annealing at $63^{\circ} \mathrm{C}$, and $45 \mathrm{~s}$ elongation at $68^{\circ} \mathrm{C}$, and a final elongation step at $72^{\circ}$ for $5 \mathrm{~min}$. The PCR products were separated by electrophoresis on a $2 \%$ agarose gel, being the 9 -allele $440 \mathrm{bp}$, and the 10 -allele $480 \mathrm{bp}$.

\section{Neuropsychological Testing}

We focused on PFC-dependent cognition, exploring components of executive functioning, specifically the following domains: impulsivity, attention, working memory, mental flexibility, planning ability, inhibitory control and adaptive behavior. A detailed description of the tests is provided in Supplementary Annex 1.

Intellectual quotient (IQ) was estimated using the Kaufman Brief Intelligence Test (K-BIT). Impulsivity was measured analyzing the relation between response times and errors in a set of tests from the non-verbal Cambridge Neuropsychological Test Automated Battery (CANTAB): Motor screening test (MOT), Simple Reaction Time (SRT), and Spatial Span Forward Recall (SSP-FR). To assess the attentional span the Digits Span Forward Recall (DSP-FR) from WAIS-III and the Spatial Span Forward (CANTAB) were administered. Working memory for visual and verbal information was evaluated with the Spatial Span Backward Recall (SSP-BR, CANTAB) and the Digits Span Backward Recall (DSP-BR) from WAIS-III, respectively. Mental flexibility was measured rating switching ability and reversal learning. Switching ability (times subject change from one semantic category to a new one) was assessed with the Semantic Fluency Word Generation Task (SFWGT, animals in $1 \mathrm{~min}$ ), and reversal learning (disengagement from inappropriate strategies when reinforcement contingencies change), with the Weigl ColorForm Sort Test (WCFST). Planning ability was measured using the Tower of London from Drexel University (ToLDx) and the Cats \& Dogs test was used to assess inhibitory control. To assess adaptive behavior, the adult version of the Adaptive Behavior Assessment System-Second Edition (ABAS-II) was used.

Finally, since early Alzheimer's disease like dementia in DS population courses with a progressive decline of the DA system and a faster degeneration of the PFC, affecting executive function (Fernandez and Reeves, 2015), we explored early dementia symptoms with the Dementia Questionnaire for People with Intellectual Disabilities (DMR).

\section{Statistical Analysis}

Descriptive analyses were carried out for sociodemographic and clinical parameters of all the participants and for all neuropsychological variables, providing measures of mean and standard deviation.

The genotypic frequency of the different polymorphisms was tested by Hardy-Weinberg equilibrium. To study the association between polymorphisms and cognition, two-way ANOVA models were fitted for each of the cognitive tests and questionnaires scores. The 2 genes were included in the models as independent factors and treated as binary variables, Val allele homozygotes vs. Met allele carriers (COMTVal158Met) and 10repeat allele homozygotes vs. 9-repeat allele carriers (VNTR$D A T 1)$, respectively. In addition, possible sources of epistatic effects on cognition were considered by assessing the interaction between COMTVal158Met and VNTR-DAT1 genotypes in each of the two-way ANOVA models.

The statistically significant associations were fixed at a significance level of 0.05 , using the model-based estimated mean differences as the effect size of interest. All statistical analyses were performed using the statistical software packages SPSS (Version 18.0; SPSS Inc., Chicago, IL, USA) and R (Version 3.1.1; The R Foundation for Statistical Computing, Vienna, Austria).

\section{RESULTS}

\section{Distribution of Polymorphisms}

Socio-demographic data and cognitive parameters for all the neuropsychological variables of the 69 participants are provided in the Supplementary Table 1.

In our study, the euploid Spanish population was used to obtain the expected frequencies for the Hardy-Weinberg equilibrium (Table 1). The proportion of COMTVal158Met allelic frequencies among the Spanish reference population $(n=93)$ was similar to the reference CEU (Utah residents with Northern and Western European ancestry), and TSI (Tuscans from Italy) HapMap populations (HapMap, n.d.); (Table 1). No data from CEU and TSI HapMap reference populations was available for the proportion of VNTR-DAT1 allelic frequencies.

In the TESDAD DS population, we found a Hardy-Weinberg disequilibrium $(\chi 2=6.47, p<0.039)$ due to an underrepresentation of the Met/Met genotype in the COMTVal158Met polymorphism, as compared to the CEU, TSI , and Spanish populations. Conversely, VNTR-DAT1 allele frequency was in the expected Hardy-Weinberg equilibrium compared to the Spanish population $(n=57)$; (Table 1$)$.

\section{VNTR-DAT1 and COMTVal158Met Effects on Cognitive and Functional Performance}

Table 2; Figures 1 and 2 show the main effects in components of executive functioning and caregiver's rating scores between VNTR-DAT1 and COMTVal158Met genotypes. The VNTRDAT1 10-repeat allele homozygotes showed statistically significant shorter response times in the CANTAB Simple Reaction Time and Spatial Span Forward Recall tasks, not linked to impulsivity since they were not accompanied by a higher number of errors. 10-repeat allele homozygotes also showed statistically significant better performance of tasks requiring mental flexibility, as measured by the SFWGT, but not by the WCFST. Taken together, these data indicate that in our DS population the 10-repeat allele homozygotes are faster responders in attentional and psychomotor tasks and have better mental flexibility. No statistically significant association was 
TABLE 1 | Distribution of COMTVal158Met and VNTR-DAT1 allele frequencies in the DS and reference populations.

\begin{tabular}{|c|c|c|c|c|c|}
\hline & & Hap map CEU population & Hap map TSI population & Spanish population & TESDAD DS population (Spanish) \\
\hline \multicolumn{6}{|c|}{ COMTVal158Met } \\
\hline & Met/Met (A/A) & $28(0.248)$ & $20(0.196)$ & $21(0.225)$ & $6(0.087)$ \\
\hline & $\mathrm{Val} / \mathrm{Met}(\mathrm{G} / \mathrm{A})$ & $52(0.460)$ & $52(0.510)$ & $45(0.484)$ & $38(0.550)$ \\
\hline & $\mathrm{Val} / \mathrm{Val}(\mathrm{G} / \mathrm{G})$ & $33(0.292)$ & $30(0.294)$ & $27(0.290)$ & $25(0.362)$ \\
\hline \multirow[t]{3}{*}{ VNTR-DAT1 } & $10 / 10$ & & & $30(0.526)$ & $35(0.507)$ \\
\hline & $10 / 9$ & & & $23(0.403)$ & $26(0.377)$ \\
\hline & $9 / 9$ & & & $4(0.070)$ & $8(0.116)$ \\
\hline
\end{tabular}

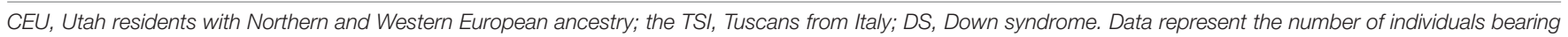
each genotype, and parenthesis account for genotypic frequencies (0-1).

TABLE 2 | VNTR-DAT1 and COMTVal158Met effects on cognitive and functional variables in the TESDAD DS population.

\begin{tabular}{|c|c|c|c|c|c|c|c|}
\hline Test & Function assessed & Polymorphism & Figure & Estimate & Standard error & $t$-value & $p$-value \\
\hline SSP-FR (CANTAB) & Mean time to response & VNTR-DAT & Figure 1A & 537 & 270 & 1.999 & 0.05 \\
\hline SRT (CANTAB) & Mean time to respond & VNTR-DAT & Figure 1B & 117 & 48 & 2.424 & 0.018 \\
\hline MOT(CANTAB) & Mean time to respond & VNTR-DAT & & 137 & 77 & 1.772 & 0.081 \\
\hline SFWGT & Switching ability: MF & VNTR-DAT & Figure 1C & -1.5 & 0.5 & -3.265 & 0.002 \\
\hline WCFST & Reversal learning: MF & VNTR-DAT & & -0.8 & 0.4 & -1.921 & 0.059 \\
\hline SFWGT & Switching ability: MF & COMTVal158Met & Figure 2A & -1.4 & 0.5 & -2.935 & 0.005 \\
\hline ABAS-Social Skills & Adaptive social behavior & COMTVal158Met & Figure 2B & 6.99 & 2.9 & 2.453 & 0.017 \\
\hline ABAS-Self-Direction & $\begin{array}{l}\text { Capacity of regulate behavior to } \\
\text { the demands of a situation. }\end{array}$ & COMTVal158Met & Figure 2C & 7.65 & 3.7 & 2.08 & 0.041 \\
\hline DMR-SOS & Social symptoms of dementia & COMTVal158Met & Figure 2D & -2.1 & 1 & -2.108 & 0.039 \\
\hline
\end{tabular}

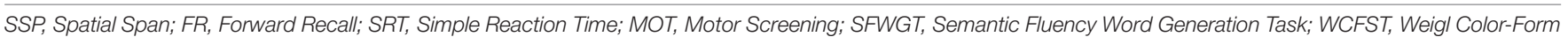

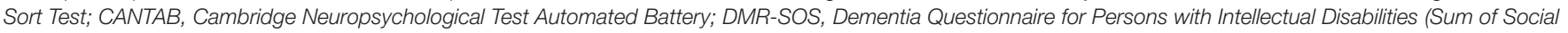

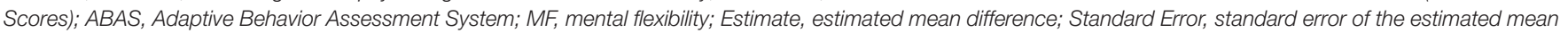

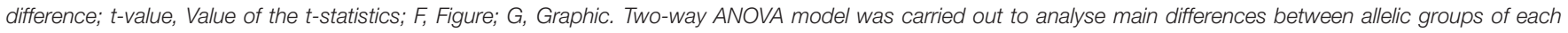
polymorphism regarding cognitive and functional outcomes.

Statistically significant associations above a significance level of 0.05 are represented as bold values.

found between the VNTR-DAT1 polymorphism and parental reports (ABAS-II and DMR).

Regarding COMTVal158Met, Met allele carriers showed no differences in response time in the CANTAB tasks but we detected the same profile as for 10-repeat allele homozygotes regarding mental flexibility that was better as assessed by the SFWGT, but not WCFST. In addition, Met allele carriers had statistically significant worse parental reports referred to adaptive behavior, specifically in the social skills and selfdirection subscales of ABAS-II, and statistically significant higher early dementia rates in the DMR social scale (DMR-SOS) than Val allele homozygotes. Thus, in our population, the Met allele carriers have a greater mental flexibility but worse adaptive behavior, along with higher social deterioration.

No statistically significant differences were found between VNTR-DAT1 or COMTVal158Met genotypes with regards to attentional span (The Digits Span Forward Recall, The Spatial Span Forward Recall from CANTAB), working memory (the Digits Span Backward Recall from WAIS and the Spatial Span Backward Recall from CANTAB), planning ability (the Tower of London from Drexel University, ToLDx), or inhibitory control (the Cats \& Dogs test).

Interactions between genotypes were considered for each cognitive and functional variable; however, no statistically significant genotype interaction between VNTR-DAT1 and COMTVal158Met polymorphisms was detected.

\section{DISCUSSION}

Since it is known that the phenotypic consequences of genetic variants are modulated by the genetic background in which they occur, we here examined the association between DA related polymorphisms VNTR-DAT1 and COMTVal158Met and interindividual differences in executive function, adaptive behavior and early symptoms of dementia in DS young adults. We show that genotypes conferring higher DA synaptic availability (Met allele carriers and 10-repeat allele homozygotes), result in improved performance in executive function tasks that require mental flexibility. We also observe worse social skills and selfdirection and earlier social symptoms of dementia in Met allele carriers but not in 10-repeat allele homozygotes.

In our DS population the genotype distribution for VNTR-DAT1 was in Hardy-Weinberg equilibrium, but the COMTVal158Met had a reduced frequency for Met allele homozygotes. Even though, there is a need of a more extensive analysis of the frequencies of the COMTVal158Met polymorphism in the DS population, and a triplet analysis 

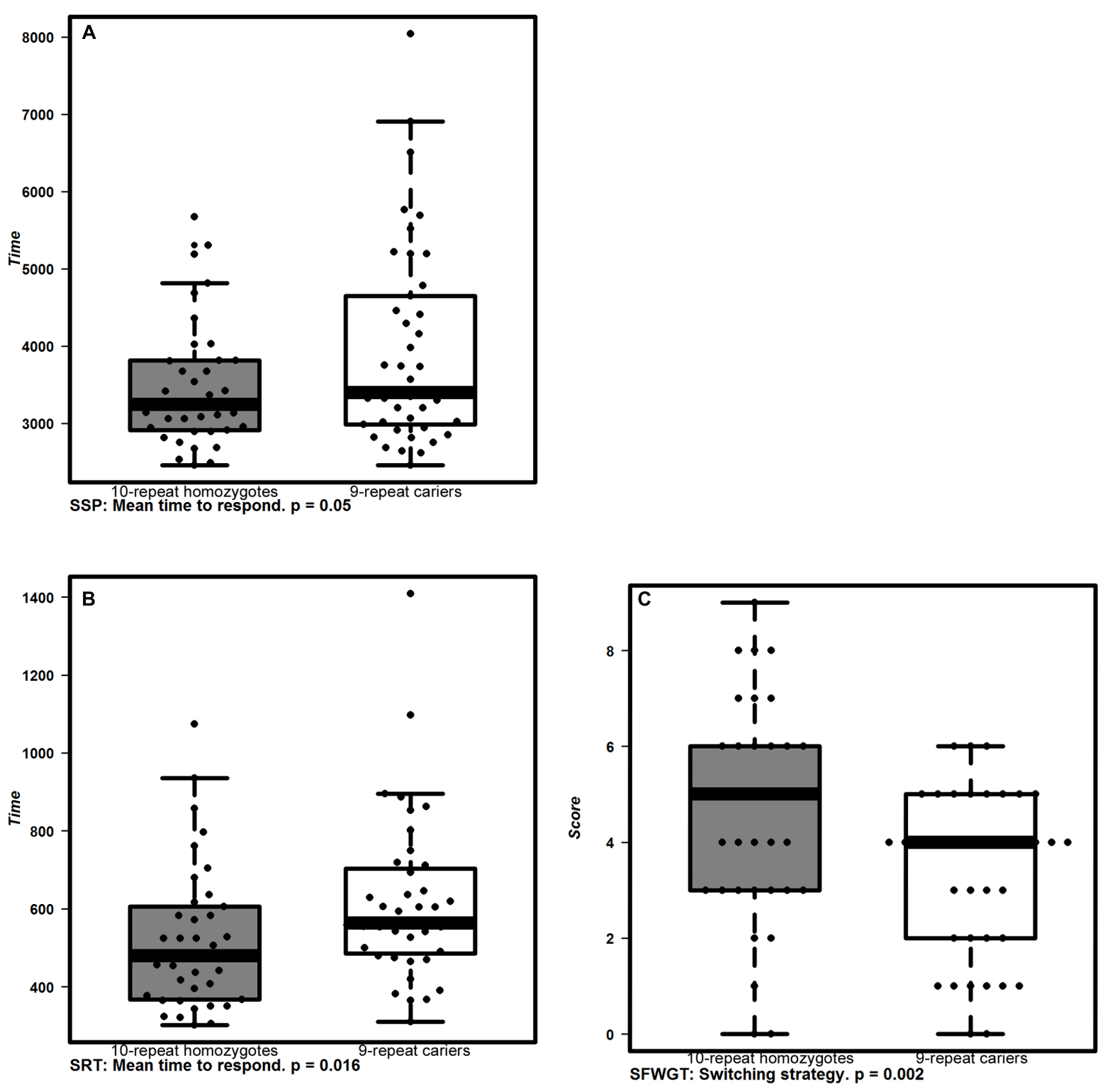

FIGURE 1 | Cognitive performance associated with VNTR-DAT1 polymorphisms. Box plots represent the cognitive performance of allelic groups of VNTR-DAT1 polymorphism. 10-repeat homozygotes (gray boxes) displayed shorter response times in CANTAB (A) SSP ( $p<0.05$; two way ANOVA) and (B) SRT tasks ( $p<0.016$; two way ANOVA), and better mental flexibility measured by (C) SFWGT (Switching strategy; $p<0.016$; two way ANOVA) than 9-repeat carriers (white boxes). The boxplots show the median and interquartile range. Dots indicate individual values.

would be required to confirm this observation, the reduced $\mathrm{Met}$ frequency in our population could be explained as a case of natural selection against the Met allele homozygotes since in the general population it has been observed that women who are carriers of the Met allele have a higher risk of miscarriage (Khadzhieva et al., 2014).

We here hypothesized that DA related polymorphisms would impact executive function in DS, but not necessarily in the same way as in euploid population, since the genetic trisomic background and the underlying cognitive deficits may influence the variants' effects. Main differences in cognitive performance between DS and age-matched controls have already been reported in our previous works (de Sola et al., 2015; Hoyo et al., 2015). Specifically, we detected a substantial deficit on executive functions, being the most impaired: semantic verbal fluency (SFWGT: total word production) and mental flexibility (SFWGT: switching ability). In our DS group, the switching ability, which allow subjects to change from one semantic category to another, is associated ( $p<0.001, r=0.689$ ) to a higher word production leading to a better performance of the task.

We here found that 10-repeat allele homozygotes were faster respondents in attentional tasks (SRT and SSP-FR), and this same 

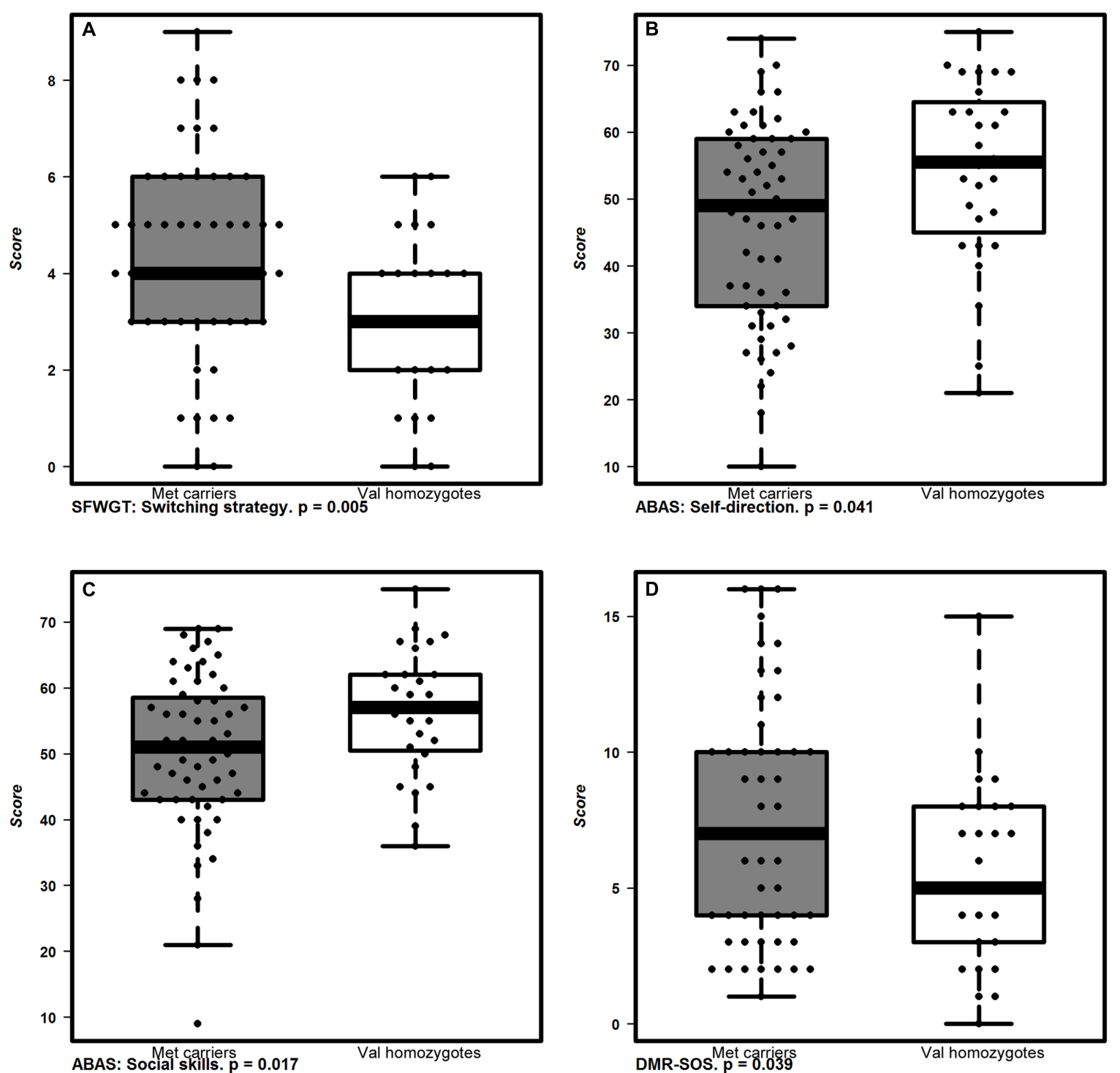

FIGURE 2 | Cognitive and functional performance associated to COMTVal158Met polymorphisms. Box plots represent the behavioral and cognitive performance of allelic groups of COMTVal158Met polymorphism. Met carriers (gray boxes) performed better in mental flexibility measured by (A) SFWGT (Switching strategy; $p$ < 0.005; two way ANOVA) and had worse parental reports in two ABAS-Il subscales: (B) ABAS-Self-Direction ( $p$ < 0.041; two way ANOVA) and (C) ABAS-Social Skills ( $p<0.017$; two way ANOVA), and also in the (D) social scale of the DMR; DMR-SOS ( $p<0.039$; two way ANOVA) than Val homozygotes (white boxes). The boxplots show the median as well as the interquartile range. Dots indicate individual values.

genotype and Met allele carriers were associated to better mental flexibility showing better switching abilities (SFWGT).

Previous studies reported that healthy subjects carrying the 10-repeat allele were more impulsive than 9-repeat allele carriers, being faster but committing more errors in a non-verbal attentional task (CPT, CANTAB; Caldú et al., 2007). Here, the faster responses of 10-repeat allele homozygotes as compared to the 9-repeat carriers could not be considered as a sign of impulsivity as there was no association between faster responses and the errors made. An association between 10-repeat allele and ADHD (Cornish et al., 2005), in which impulsivity is a core characteristic
(Winstanley et al., 2006), has also been demonstrated, being the hyperactive/impulsive-ADHD-type linked with the 10repeat allele (Waldman et al., 1998). Although there is a high ADHD prevalence in DS population (Ekstein et al., 2011), in our study there was no ADHD comorbidity nor signs of impulsivity at a group level, so we could not determine such association.

The association between Met allele carriers and better mental flexibility is in agreement with previous studies in general population in which subjects with the low-activity Met allele were associated to fewer perseverative errors in a mental flexibility task (Malhotra et al., 2002). 
Taken together, our results indicate that 10-repeat allele homozygotes and Met allele carriers in our DS population have better mental flexibility. Interestingly, a reduction of the levels of DA in the frontal cortex of fetal DS brains has been observed (Whittle et al., 2007) that is associated with dysfunctional neuronal development in DS. Therefore, even though we have not directly measured PFC DA availability, Met allele carriers and 10-repeat allele homozygotes, which are supposed to have higher frontal DA availability, could be compensating PFC dependent executive functions (Rowe et al., 2006).

Since executive skills are strongly associated with adaptive behavior (ABAS-II), we explored how these polymorphisms were linked to adaptive behavior skills. Our results showed differences in parental-reports between COMTVal158Met genotypes but not between VNTR-DAT1 genotypes. Met allele carriers showed worse adaptive behavior, concretely worse social skills and selfdirection. Self-direction is a subscale of the ABAS-II which measures the executive control in daily living. Social abilities are also mediated by executive function, which helps the subject to adapt to different social situations regulating emotion (Campbell et al., 2015). Previous studies in general population have also find a positive association between the $\mathrm{Val}$ allele and social skills (Walter et al., 2011). Our results showed that the Met allele is conferring an advantage in cognition (mental flexibility) but penalizing for adaptive behavior skills, which has been shown either in healthy and disease conditions (Mier et al., 2009).

Finally, Met allele carriers were also associated to worse scores in the social scale of the DMR (DMR-SOS) than high-activity $\mathrm{Val}$ allele homozygotes. Conversely, some reports suggest that increased synaptic DA catabolism promotes the neurodegeneration within DA-innervated brain regions and thus, progressive cognitive and behavioral decline associated with AD (Gennatas et al., 2012). However, even though the Met allele (increasing DA availability) may confer resistance to $\mathrm{AD}$ (Gennatas et al., 2012), in our sample Met allele carriers did not show differences in the general DMR score. Thus, we interpret this result as an association between Met allele and social deterioration (DMR-SOS), instead of a link with early social dementia symptoms, since Met allele carriers also showed worse adaptive social behavior (ABAS-II).

In DS, one study has reported that the DRD4 7-repeat allele was related to behavioral and executive functions difficulties (Mason et al., 2015). We here did not explore these variants and thus, possible interactions of our polymorphisms and the DRD4 gene variants could also be contributing to the detected phenotypes. Other genotypes examined seem to little contribute (genetic polymorphisms in the dopaminergic and serotonergic system the DRD4 exon 3 VNTR and the 5-HTTLPR) to the cognitive dysfunctions observed in DS (Das Bhowmik et al., 2008).

\section{Limitations}

First, even though the sample size of this study is larger than other single-gene association studies in people with DS (Mason et al., 2015), a larger sample size would have been ideal to confirm our results. However, limited sample sizes are expected in most studies conducted in special populations. Secondly, we did not have a comparison group, although our effects were similar to typically developing samples reported in the literature. Future studies should explore whether COMTVal158Met and VNTR-DAT1 effects on executive function and behavior in adults with DS are additive or enhanced relative to effects in typically developing individuals. Finally, the large number of statistical tests carried out may increase the probability of Type1 errors. Nonetheless, since this is rather an exploratory than a confirmatory study, no correction to control a family-wise significance level of 0.05 has been applied in order not to increase the probability of Type- 2 .

\section{CONCLUSION}

Our study has evaluated whether allelic variants of specific nontrisomy 21 genes (COMTVal158Met and VNTR-DAT1) could contribute to explain cognitive and behavioral variability in DS phenotype. While the phenotype is most likely due to a subtle increase in gene dosage, how genetic variants out of the chromosome 21 and related to DA transmission would affect variability in prefrontal cognition in an aneuploidy genomic context is little explored. Since it is known that the phenotypic consequences of genetic variants are modulated by the genetic background in which they occur, we here explore whether COMTVal158Met and VNTR-DAT1 variants interact with the trisomic genetic background to influence gene expression, and how this in turn mediates Down syndrome phenotypes. Our results suggest that genotypes conferring higher DA availability as for Met allele carriers and 10-repeat allele homozygotes result in improved mental flexibility. With respect to parental reports, worse rates in adaptive behavior regarding social skills and self-direction were found only in the Met allele carriers, along with higher social deterioration. We conclude that genetic variations related to DA transmission may have an impact on the cognitive and behavioral DS phenotype similar to the general population. Nevertheless, further longitudinal studies are needed to confirm our results and determine whether these differences are unaffected or significantly enhanced by Trisomy 21 on molecular (epigenetic) and cognitive/behavioral and functional levels.

\section{ETHICAL STANDARDS}

The authors assert that all procedures contributing to this work comply with the ethical standards of the relevant national and institutional committees on human experimentation and with the Helsinki Declaration of 1975, as revised in 2008. The study was conducted in accordance with the Declaration of Helsinki and Spanish laws concerning data privacy. The study was conducted in accordance with the Declaration of Helsinki and Spanish laws concerning data privacy. The protocol was approved by the Ethical Committee of the Parc de Salut Mar of Barcelona (CEIC-PSMAR). Upon arrival at the research centre (Hospital del Mar Medical Research Institute-IMIM), participants, parents and legal guardians (in case of legal incapacitation) were informed 
of the ensuing protocol and they gave their written informed consent before participating.

\section{MEMBERS OF THE TESDAD STUDY GROUP}

Aida Cuenca-Royo, Hospital del Mar Medical Research Institute, Barcelona, Spain, Neuropsychologist, Site Investigator; Alessandro Principe, Hospital del Mar Medical Research Institute, Barcelona, Spain, Neurophysiology Section, Site Investigator; Bessy Benejam, Catalan Foundation of Down Syndrome, FCSD, Barcelona, Spain, Neuropsychologist, site investigator; Ester Civit, Hospital del Mar Medical Research Institute, Barcelona, Spain, Site Investigator; Gimena Hernandez, Hospital del Mar Medical Research Institute, Barcelona, Spain, Site Investigator; Gonzalo Sánchez-Benavides, Hospital del Mar Medical Research Institute, Barcelona, Spain, Neuropsychologist, Site Investigator; Henri Bléhaut, Jérôme Lejeune Foundation, Paris, France, Site investigator; Iván Dueñas, Hospital del Mar Medical Research Institute, Barcelona, Spain; Jesús Pujol, Neurovoxel, Barcelona, Spain, neurologist/neuroimaging, Site Investigator; Joan Rodríguez, Hospital del Mar Medical Research Institute, Barcelona, Spain, Study Coordinator; Jordi Peña-Casanova, Hospital del Mar Medical Research Institute, Barcelona, Spain, Dementia Section, Site Investigator; Josep $\mathbf{M}^{\mathrm{a}}$ Espadaler, Hospital del Mar Medical Research Institute, Barcelona, Spain, Neurophysiology Section, Site Investigator; Judit Sánchez, Neuropsychlogist, Feskits, Barcelona, Spain, Site Investigator; Katy Trias, Catalan Foundation of Down Syndrome, FCSD, Barcelona, Spain, Site Investigator; Klaus Langohr, Polytechnics University, Barcelona, Spain, Statistician; Laia Roca, Hospital del Mar Medical Research Institute, Barcelona, Spain, Study Manager; Laura Blanco, Hospital del Mar Medical Research Institute, Barcelona, Spain, Neuropsychologist, Site Investigator; Laura del Hoyo, Hospital del Mar Medical Research Institute, Barcelona, Spain, Neuropsychologist, Site Investigator; Laura Xicota, Hospital del Mar Medical Research Institute, Barcelona, Spain, Site Investigator; Magí Farré, Hospital del Mar Medical Research Institute, Barcelona, Spain, co-PI; Mara Dierssen, Centre for Genomic Regulation- CRG of Barcelona, co-PI; Rafael de la Torre, Hospital del Mar Medical Research Institute, Barcelona, Spain, PI; Rut Freixas, Centre for Genomic Regulation- CRG of Barcelona, Site Investigator; Sebastià Videla, Catalan Foundation of Down Syndrome, FCSD, Barcelona, Spain, site investigator; Silvina Catuara-Solarz, Centre for Genomic Regulation- CRG

\section{REFERENCES}

Barnett, J. H., Xu, K., Heron, J., Goldman, D., and Jones, P. B. (2011). Cognitive effects of genetic variation in monoamine neurotransmitter systems: a population-based study of COMT, MAOA, and 5HTTLPR. Am. J. Med. Genet. B Neuropsychiatr. Genet. 156, 158-167. doi: 10.1002/ajmg.b. 31150

Caldú, X., Vendrell, P., Bartrés-Faz, D., Clemente, I., Bargalló, N., Jurado, M. A., et al. (2007). Impact of the COMT Val108/158 Met and DAT genotypes of Barcelona, Site Investigator; Susana de Sola, Hospital del Mar Medical Research Institute, Barcelona, Spain, Neuropsychologist, Site Investigator; Valérie Legout, Jérôme Lejeune Foundation, Paris, France, Site Investigator.

\section{AUTHOR CONTRIBUTIONS}

LdH conceived and design this study, carried out the neuropsychological assessment, collected and analyzed the data, interpreted the results, and wrote the manuscript. LX and JR-M carried out the genotyping; LX interpreted the results, and contributes to manuscript preparation. KL reviewed and carried the statistical analysis, and manuscript preparation. SS, AC-R, and GS-B carried out the neuropsychological assessment. LdH and JR carried out the medical explorations, and coordinate the experiments. MF, MD, and RT conceived the study design and reviewed the manuscript. All authors read and approved the manuscript.

\section{FUNDING}

This work was supported by grants, donations and agreements from Fondation Jérôme Lejeune (Paris, France), Instituto de Salud Carlos III FEDER, (PI11/00744), MINECO (SAF201019434 and SAF2013-49129-C2-1-R), EU (Era Net Neuron PCIN-2013-060), DIUE de la Generalitat de Catalunya (SGR 2009/1450, 2014SGR 680, 2014 SGR 464, SGR 2009/718, and SGR 2014/1125). LdH is recipient of a pre-doctoral fellowship from ISCIII [FI12/00319]. CRG is Center of Excellence Severo Ochoa SEV-2012-0208.

\section{ACKNOWLEDGMENTS}

We are in debt with the support of the families that participated in the study and in particular with the contribution of the Catalan Down's Syndrome Foundation (Barcelona, Spain) and Down España (Madrid, Spain) that made possible to perform the study.

\section{SUPPLEMENTARY MATERIAL}

The Supplementary Material for this article can be found online at: http://journal.frontiersin.org/article/10.3389/fnbeh. 2016.00193

on prefrontal function in healthy subjects. Neuroimage 37, 1437-1444. doi: 10.1016/j.neuroimage.2007.06.021

Campbell, L. E., McCabe, K. L., Melville, J. L., Strutt, P. A., and Schall, U. (2015). Social cognition dysfunction in adolescents with 22q11.2 deletion syndrome (velo-cardio-facial syndrome): relationship with executive functioning and social competence/functioning. J. Intellect. Disabil. Res. 59, 845-859. doi: $10.1111 /$ jir.12183

Cornish, K. M., Manly, T., Savage, R., Swanson, J., Morisano, D., Butler, N., et al. (2005). Association of the dopamine transporter (DAT1) 10/10-repeat genotype 
with ADHD symptoms and response inhibition in a general population sample. Mol. Psychiatry 10, 686-698. doi: 10.1038/sj.mp.4001641

Cuyàs, E., Verdejo-García, A., Fagundo, A. B., Khymenets, O., Rodríguez, J., Cuenca, A., et al. (2011). The influence of genetic and environmental factors among MDMA users in cognitive performance. PLoS ONE 6:e27206. doi: 10.1371/journal.pone.0027206

Das Bhowmik, A., Dutta, S., Sinha, S., Chattopadhyay, A., and Mukhopadhyay, K. (2008). Lack of association between down syndrome and polymorphisms in dopamine receptor D4 and serotonin transporter genes. Neurochem. Res. 33, 1286-1291. doi: 10.1007/s11064-007-9581-9

de Sola, S., de la Torre, R., Sanchez-Benavides, G., Benejam, B., Cuenca-Royo, A., del Hoyo, L., et al. (2015). A new cognitive evaluation battery for down syndrome and its relevance for clinical trials. Front. Psychol. 6:708. doi: 10.3389/fpsyg.2015.00708

Edgin, J. O., Mason, G. M., Spanò, G., Fernández, A., and Nadel, L. (2012). Human and mouse model cognitive phenotypes in down syndrome: implications for assessment. Prog. Brain Res. 197, 123-151. doi: 10.1016/B978-0-444-54299$1.00007-8$

Ekstein, S., Glick, B., Weill, M., Kay, B., and Berger, I. (2011). Down syndrome and attention-deficit/hyperactivity disorder (ADHD). J. Child Neurol. 26, 1290 1295. doi: $10.1177 / 0883073811405201$

Fernandez, F., and Reeves, R. H. (2015). Assessing cognitive improvement in people with down syndrome: important considerations for drug-efficacy trials. Handb. Exp. Pharmacol. 228, 335-380. doi: 10.1007/978-3-319-16522-6_12

Gennatas, E. D., Cholfin, J. A., Zhou, J., Crawford, R. K., Sasaki, D. A., Karydas, A., et al. (2012). COMT Val158Met genotype influences neurodegeneration within dopamine-innervated brain structures. Neurology 78, 1663-1669. doi: 10.1212/WNL.0b013e3182574fal

Grieco, J., Pulsifer, M., Seligsohn, K., Skotko, B., and Schwartz, A. (2015). Down syndrome: cognitive and behavioral functioning across the lifespan. Am. J. Med. Genet. C Semin. Med. Genet. 169, 135-149. doi: 10.1002/ajmg.c.31439

HapMap Project (n.d.). Available at: http://hapmap.ncbi.nlm.nih.gov/ Accessed[August, 2016].

Hoyo, L. D., Xicota, L., Sánchez-Benavides, G., Cuenca-Royo, A., de Sola, S., Langohr, K., et al. (2015). Semantic verbal fluency pattern, dementia rating scores and adaptive behavior correlate with plasma $A \beta 42$ concentrations in down syndrome young adults. Front. Behav. Neurosci. 9:301. doi: 10.3389/fnbeh.2015.00301

Khadzhieva, M. B., Lutcenko, N. N., Volodin, I. V., Morozova, K. V., and Salnikova, L. E. (2014). Association of oxidative stress-related genes with idiopathic recurrent miscarriage. Free Radic. Res. 48, 534-541. doi: 10.3109/10715762.2014.891735

Korenberg, J. R., Chen, X. N., Schipper, R., Sun, Z., Gonsky, R., Gerwehr, S., et al. (1994). Down syndrome phenotypes: the consequences of chromosomal imbalance. Proc. Natl. Acad. Sci. U.S.A. 91, 4997-5001. doi: 10.1073/pnas.91.11.4997

Koutsilieri, E., Riederer, P., du Plessis, S., and Scheller, C. (2014). A short review on the relation between the dopamine transporter 10/10-repeat allele and ADHD: implications for HIV infection. Atten. Defic. Hyperact. Disord. 6, 203-209. doi: 10.1007/s12402-014-0134-1

Lachman, H. M., Papolos, D. F., Saito, T., Yu, Y.-M., Szumlanski, C. L., and Weinshilboum, R. M. (1996). Human catechol-O-methyltransferase pharmacogenetics: description of a functional polymorphism and its potential application to neuropsychiatric disorders. Pharmacogenetics 6, 243-250. doi: 10.1097/00008571-199606000-00007

Lumbreras, M., Baamonde, C., Martínez-Cué, C., Lubec, G., Cairns, N., Sallés, J., et al. (2006). Brain $G$ protein-dependent signaling pathways in down syndrome and Alzheimer's disease. Amino Acids 31, 449-456. doi: 10.1007/s00726-005$0272-z$

Malhotra, A. K., Kestler, L. J., Mazzanti, C., Bates, J. A., Goldberg, T., and Goldman, D. (2002). A functional polymorphism in the COMT gene and performance on a test of prefrontal cognition. Am. J. Psychiatry 159, 652-654. doi: 10.1176/appi.ajp.159.4.652

Mason, G. M., Spanó, G., and Edgin, J. (2015). Symptoms of attentiondeficit/hyperactivity disorder in down syndrome: effects of the dopamine receptor D4 gene. Am. J. Intellect. Dev. Disabil. 120, 58-71. doi: 10.1352/19447558-120.1.58

Meyer-Lindenberg, A., Kohn, P. D., Kolachana, B., Kippenhan, S., McInerneyLeo, A., Nussbaum, R., et al. (2005). Midbrain dopamine and prefrontal function in humans: interaction and modulation by COMT genotype. Nat. Neurosci. 8, 594-596. doi: 10.1038/nn1438

Mier, D., Kirsch, P., and Meyer-Lindenberg, A. (2009). Neural substrates of pleiotropic action of genetic variation in COMT: a meta-analysis. Mol. Psychiatry 15, 918-927. doi: 10.1038/mp.2009.36

Miller, E. K., and Cohen, J. D. (2001). An integrative theory of prefrontal cortex function. Annu. Rev. Neurosci. 24, 167-202. doi: 10.1146/annurev.neuro.24.1.167

Risser, D., Lubec, G., Cairns, N., and Herrera-Marschitz, M. (1997). Excitatory amino acids and monoamines in parahippocampal gyrus and frontal cortical pole of adults with down syndrome. Life Sci. 60, 1231-1237. doi: 10.1016/S00243205(97)00067-2

Rowe, J., Lavender, A., and Turk, V. (2006). Cognitive executive function in down's syndrome. Br. J. Clin. Psychol. 45(Pt 1), 5-17. doi: 10.1348/014466505X29594

Schneider, C., Risser, D., Kirchner, L., Kitzmüller, E., Cairns, N., Prast, H., et al. (1997). Similar deficits of central histaminergic system in patients with down syndrome and Alzheimer disease. Neurosci. Lett. 222, 183-186. doi: 10.1016/S0304-3940(97)13379-1

Shumay, E., Chen, J., Fowler, J. S., and Volkow, N. D. (2011). Genotype and ancestry modulate brain's DAT availability in healthy humans. PLOS ONE 6:e22754. doi: 10.1371/journal.pone.0022754

van de Giessen, E. M., de Win, M. M. L., Tanck, M. W. T., van den Brink, W., Baas, F., and Booij, J. (2009). Striatal dopamine transporter availability associated with polymorphisms in the dopamine transporter gene SLC6A3. J. Nucl. Med. 50, 45-52. doi: 10.2967/jnumed.108. 053652

Verdejo-García, A., Fagundo, A. B., Cuenca, A., Rodriguez, J., Cuyás, E., Langohr, K., et al. (2013). COMT val158met and 5-HTTLPR genetic polymorphisms moderate executive control in cannabis users. Neuropsychopharmacology 38, 1598-1606. doi: 10.1038/npp.2013.59

Waldman, I. D., Rowe, D. C., Abramowitz, A., Kozel, S. T., Mohr, J. H., Sherman, S. L., et al. (1998). Association and linkage of the dopamine transporter gene and attention-deficit hyperactivity disorder in children: heterogeneity owing to diagnostic subtype and severity. Am. J. Hum. Genet. 63, 1767-1776. doi: $10.1086 / 30213$

Walter, N. T., Markett, S. A., Montag, C., and Reuter, M. (2011). A genetic contribution to cooperation: dopamine-relevant genes are associated with social facilitation. Soc. Neurosci. 6, 289-301. doi: 10.1080/17470919.2010.527169

Whittle, N., Sartori, S. B., Dierssen, M., Lubec, G., and Singewald, N. (2007). Fetal down syndrome brains exhibit aberrant levels of neurotransmitters critical for normal brain development. Pediatrics 120, e1465-e1471. doi: 10.1542/peds.2006-3448

Winstanley, C. A., Eagle, D. M., and Robbins, T. W. (2006). Behavioral models of impulsivity in relation to ADHD: translation between clinical and preclinical studies. Clin. Psychol. Rev 26, 379-395. doi: 10.1016/j.cpr.2006.01.001

Witte, A. V., and Flöel, A. (2012). Effects of COMT polymorphisms on brain function and behavior in health and disease. Brain Res. Bull. 88, 418-428. doi: 10.1016/j.brainresbull.2011.11.012

Wonodi, I., Hong, L. E., Stine, O. C., Mitchell, B. D., Elliott, A., Roberts, R. C., et al. (2009). Dopamine transporter polymorphism modulates oculomotor function and DAT1 mRNA expression in schizophrenia. Am. J. Med. Genet. B Neuropsychiatr. Genet. 150B, 282-289. doi: 10.1002/ajmg.b.30811

Conflict of Interest Statement: The authors declare that the research was conducted in the absence of any commercial or financial relationships that could be construed as a potential conflict of interest.

The reviewer DM and handling Editor declared their shared affiliation, and the handling Editor states that the process nevertheless met the standards of a fair and objective review.

Copyright (๐) 2016 del Hoyo, Xicota, Langohr, Sánchez-Benavides, de Sola, CuencaRoyo, Rodriguez, Rodríguez-Morató, Farré, Dierssen, de la Torre and the TESDAD Study Group. This is an open-access article distributed under the terms of the Creative Commons Attribution License (CC BY). The use, distribution or reproduction in other forums is permitted, provided the original author(s) or licensor are credited and that the original publication in this journal is cited, in accordance with accepted academic practice. No use, distribution or reproduction is permitted which does not comply with these terms. 